[6] A. Carnevale and U. C. Paek, "Modal structure of an MCVD optical waveguide fiber," Bell Syst. Tech. J., vol. 62, pp. 1415-1431, 1983.

[7] M. D. Feit and J. A. Fleck, Jr., "Mode properties and dispersion for two optical fiber-index profiles by the propagating beam method," Appl. Opt., vol. 19, pp. 3140-3150, 1980.

[8] P. M. Rodhe, "Calculation of scalar modes of optical fibres with circular, symmetric cross-sections," Instit. Opt. Res., Stockholm, Sweden, Tech. Rep. TR117, 1982.

[9] R. A. Sammut, C. D. Hussey, J. D. Love, and A. W. Snyder, "Modal analysis of polarization effects in weakly-guiding fibres," IEE Proc., vol. $128 \mathrm{H}$, pp. 173-187, 1981.

[10] A. Papoulis, Probability, Random Variables, and Stochastic Processes. New York: McGraw-Hill, 1965, ch. 4-7.

[11] D. Marcuse, Theory of Dielectric Optical Waveguides. New York: Academic Press, 1974, ch. 5.

[12] G. W. Day, Measurement of Optical Fiber Bandwidth in the Frequency Domain, NBS TN-1046, Washington: U.S. Government Printing Office, 1981.

[13] P. M. Rodhe, "Intramodal part of the transfer function for an optical fiber," pp. 154-158, this issue.

[14] S. D. Personick, "Baseband linearity and equalization in fiber optical digital communication systems," Bell Syst. Tech. J., vol. 52, pp. 1175-1194, 1973.

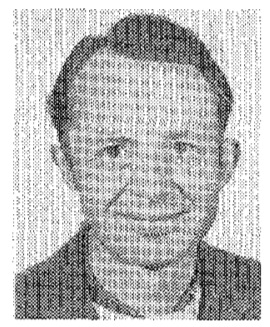

Peder M. Rodhe was born in Stockholm, Sweden, on May 13, 1945. He received the M.S. degree in physics from the University of Stockholm, in 1973 and the Lic.Techn. and Ph.D. degrees in optics and wave theory from the Royal Institute of Technology, Stockholm, in 1982 and 1984 , respectively.

From 1975 to 1979 he was engaged in research and development of optical fibers at Ericsson in Stockholm. After that he joined the Fiber Measurement Group at Sieverts Kabelverk AB, Sundbyberg and from 1980 to 1982 he also was an assistant at the Institute of Optical Research, Stockholm. On a fellowship from the Wallenberg Foundation he became a guest worker at the National Bureau of Standards, Boulder, CO, from 1982 to 1984, also joining the University of Colorado as a Visiting Research Associate. Very recently, he obtained a position as an engineering scientist at the Photonics Research Group at AMP Incorporated, Harrisburg, PA.

\title{
Intramodal Part of the Transfer Function for an Optical Fiber
}

\author{
PEDER M. RODHE
}

\begin{abstract}
Intramodal contributions in measurements of optical-fiber bandwidth are investigated theoretically and experimentally in the quasimonochromatic case. A relation is established between the intramodal transfer function and a possibly non-Gaussian source spectrum, which may also vary with modulation frequency. By considering the latter variation in particular, we are able to predict the intramodal length dependence and show how it may deviate from that of a conventional approach.
\end{abstract}

\section{INTRODUCTION}

$T^{0}$ O OBTAIN THE intermodal part (due to the different modal group delays) of the baseband transfer function for an optical fiber or a chain of jointed fibers, it will first be necessary to extract the intramodal part (related to the source). This problem is usually addressed by assuming a stationary, Gaussian form for the wavelength spectrum of the light source and a certain material dispersion for the fiber [1]. It is then easy to deconvolve the resulting, Gaussian, intramodal impulse response from the total impulse response [2], [3]. In the case of narrow-band sources and short fibers

Manuscript received March 26, 1984; revised September 4, 1984.

The author was with the National Bureau of Standards, Electromagnetic Division, Optical Electronic Metrology Group, Boulder, CO 80303, on leave from SI Sieverts Kabelverk AB, Sundbyberg, Sweden. He is now with the Photonics Research Group, AMP Inc., Harrisburg, PA. $(\sim 1 \mathrm{~km})$ it may be argued that the intramodal contribution will be negligible [2]; however, this may not be satisfactory in applications involving high baseband frequencies.

In this paper, we discuss quasimonochromatic source spectra, which may vary with modulation, even exhibiting a chirping behavior [4]. We apply this to a particular case of a continuously emitting, sinusoidally modulated semiconductor laser, intended for bandwidth characterization of single fibers as well as longer fiber chains. Conventionally, intramodal bandwidth is expected to vary inversely proportionally to fiber length; however, as we illustrate here, this will not be valid if appreciable spectral variation occurs.

\section{Theoretical BACKground}

\section{A. The Intramodal Transfer Function}

We base our derivation of the intramodal transfer function on a general model for propagation in an optical fiber of modulated, monochromatic light power. It should here be appropriate to adopt the results of time-dependent coupled power theory [5]. An intermodal transfer function may thus be formally expressed by

$$
\begin{array}{r}
T_{\text {inter }}(\lambda, z, \omega)=T_{\text {inter }}\left[z, \omega, \tau_{\mu}(\lambda), a_{\mu}(\lambda), h_{\mu \nu}(\lambda), \cdots\right], \\
\mu, \nu=1,2,3, \cdots, M, \mu \neq \nu
\end{array}
$$


that is, such a function will depend on the parameters $\tau_{\mu}, a_{\mu}$ and $h_{\mu \nu}$ of all the $M$ guided modes, as well as on fiber length $z$ and angular modulation frequency $\omega . \tau_{\mu}$ is here the group delay per unit fiber length and $a_{\mu}$ is the power attenuation of mode $\mu$, while $h_{\mu \nu}$ is the power coupling coefficient for modes $\mu$ and $\nu$.

As indicated in (1), these parameters should all depend on wavelength $\lambda$. For the further discussion, we make the usual approximation, that the light source is quasimonochromatic, which means that its spectral width is much less than $\lambda_{c}$, the center wavelength of its power spectrum. Accordingly, over the narrow spectral band in question, it is reasonable to assume $\tau_{\mu}$ to be the only mode parameter having a wavelength variation important for our derivation.

It is convenient to put $\tau_{\mu}$ in the form

$$
\tau_{\mu}(\lambda) \equiv \tau_{\mu}\left(\lambda_{c}\right)+\int_{\lambda_{c}}^{\lambda} \delta_{\mu}(x) d x
$$

where

$$
\delta_{\mu}(\lambda)=\frac{d \tau_{\mu}(\lambda)}{d \lambda} .
$$

Since we are mostly interested in high-bandwidth fibers [2], $\delta_{\mu}$ can be assumed nearly mode independent, thus constituting an effective material dispersion coefficient. Moreover, this coefficient can be regarded as practically constant within a narrow wavelength band. Hence, we may put $\delta_{\mu} \simeq \delta_{\mu}\left(\lambda_{c}\right) \equiv \delta_{c}$, so that (2) converts into

$$
\tau_{\mu}(\lambda)=\tau_{\mu}\left(\lambda_{c}\right)+\left(\lambda-\lambda_{c}\right) \delta_{c}
$$

A general form for $T_{\text {inter }}$ can indeed be derived from timedependent coupled power theory. This together with the assumptions given above and (4) allow us to write (1) as

$$
T_{\text {inter }}(\lambda, z, \omega)=T_{\text {inter }}\left(\lambda_{c}, z, \omega\right) \exp \left[-i\left(\lambda-\lambda_{c}\right) \delta_{c} \omega z\right]
$$

(see Appendix A) where $i$ is the imaginary unit. It should thus be sufficient to consider the intermodal transfer function at the center wavelength $\lambda_{c}$. The second term of (4) will contribute to this function a phase shift which goes linearly with fiber length, modulation frequency, and wavelength. A total transfer function may be obtained by integrating weighted contributions of the form of (5) with respect to wavelength

$$
\begin{aligned}
T_{\text {tot }}(z, \omega)= & T_{\text {inter }}\left(\lambda_{c}, z, \omega\right) \int_{0}^{\infty} s(\lambda, \omega) \\
& \cdot \exp \left[-i\left(\lambda-\lambda_{c}\right) \delta_{c} \omega z\right] d \lambda .
\end{aligned}
$$

To allow for variations in connection with modulation of the light source, we have introduced in (6) a weighting function $s(\lambda, \omega)$, which may be complex, depending on modulation frequency as well as wavelength. In addition, this function should be normalized according to

$$
\int_{0}^{\infty} s(\lambda, \omega) d \lambda=1
$$

In physical terms, $s(\lambda, \omega)$ thus describes the baseband variations of each component of the power spectrum of the source. For $\omega=0, s$ is the usual stationary power spectrum.

The intramodal part of the total transfer function can now be defined by the integral in (6),

$$
T_{\text {intra }}(z, \omega)=\int_{0}^{\infty} s(\lambda, \omega) \exp \left[-i\left(\lambda-\lambda_{c}\right) \delta_{c} \omega z\right] d \lambda .
$$

It may be useful here to substitute $\lambda \rightarrow \gamma=\lambda-\lambda_{c}$ and put $\Omega=\delta_{c} \omega z$. Equation (8) can then be written in the form

$$
T_{\mathrm{intra}}(\Omega, \omega)=\int_{-\infty}^{\infty} s(\gamma, \omega) \exp (-i \gamma \Omega) d \gamma
$$

The lower integration limit is allowed, since $s(\gamma, \omega)$ is expected to take appreciable values only very near $\gamma=0$. Equation (9) shows, that for each fixed $\omega, T_{\text {intra }}$ and $s$ form a Fourier pair in the domains of $\Omega$ and $\gamma$, respectively. Accordingly, a Fourier expansion of $s$ becomes

$$
s(\gamma, \omega)=\frac{1}{2 \pi} \int_{-\infty}^{\infty} T_{\mathrm{intra}}(\Omega, \omega) \exp (i \gamma \Omega) d \Omega .
$$

In the particular case of a source spectrum invariant to modulation, we find from (8) that $T_{\text {intra }}$ depends only on the product of modulation frequency and fiber length, meaning for example that the intramodal $-3-\mathrm{dB}$ bandwidth becomes inversely proportional to length, a condition which is usually assumed in practice. Also note in this case, that if $s$ is approximated by a Gaussian function, obtained for example by a fitting procedure, there is a simple relation between $B_{\text {intra }}(z)$, the $-3-\mathrm{dB}$ bandwidth (in hertz), and $w_{s}$, the full width at half maximum (FWHM) of $s$

$$
B_{\text {intra }}(z)=\left|2 \ln 2 / \pi w_{s} \delta_{c} z\right| .
$$

\section{B. Resolution Effects in Spectral Measurements}

In measuring the irradiance from a general source, we may show that, in the limit of long measuring time, the contribution from each individual wavelength component can be treated independently. This should also hold for such components after having passed through a monochromator spectrometer, including a grating or other spatially dispersive devices [6].

Accordingly, we may consider a monochromator as a filter of tunable center wavelength $\lambda_{t c}$, having a passband described by the transmittance function $u(\eta)$, where $\eta=\lambda-\lambda_{t c}$. While we keep $\omega$ constant, we can measure the wavelength variation of $s(\lambda, \omega)$ through the filter. The result will be a convolution between $u$ and $s$.

$$
v(\lambda, \omega)=\int_{-\infty}^{\infty} u(\lambda-x) s(x, \omega) d x .
$$

In accordance with (12) we may also write

$$
\sigma_{v}=\left(\sigma_{u}^{2}+\sigma_{s}^{2}\right)^{1 / 2}
$$

which relates the rms spectral widths of the functions $s, u$, and $v$. We may decide that the effects of a finite resolution (a finite 
rms width of $u$ ) are negligible whenever $\sigma_{u}$ is less than a certain fraction of $\sigma_{s}$. Since the exact form of the function $u$ is not usually known, we might approximate it by a Gaussian. For the purpose of estimating the effects of $u$ in measurements, it might even be useful to approximate $s$ and $v$ in the same way. Equation (13) then also relates the FWHM's of these approximated functions.

\section{Sampling of Spectral Data}

In practice, the resolution effects above can often be neglected. Instead of (8) we may in that case consider the approximate formula

$$
\begin{aligned}
T_{\text {intra }}(z, \omega)= & \sum_{n=1}^{N} s_{n}(\omega) \exp \left[i \theta_{n}(\omega)\right] \\
& \cdot \exp \left(-\operatorname{ind}_{\lambda} \delta_{c} \omega z\right) / \sum_{n=1}^{N} s_{n}(\omega) \\
& \cdot \exp \left[i \theta_{n}(\omega)\right]
\end{aligned}
$$

where

$$
d_{\lambda}=\lambda_{n+1}-\lambda_{n}>0 .
$$

For any fixed $\omega, s_{n}$, and $\theta_{n}$ are the amplitude and relative phase of $s(\lambda, \omega)$, respectively, sampled at $N$ evenly spaced positions within a properly chosen wavelength interval (there and nowhere else should $s$ take appreciable values). Equation (14) may be regarded as a normalized Fourier series with a fundamental period of $2 \Pi / d_{\lambda}$. Referring to the sampling theorem [7],(14) will hold exactly if the true $s$ is bandlimited, that is, if $T_{\text {intra }}$ is zero outside the two Nyquist frequencies, $\delta_{c} \omega z= \pm \pi / d_{\lambda}$. We may expect this to hold reasonably well in our particular case, which involves a multimode CW semiconductor laser source. Such sources usually have nearly discrete spectra, resulting from the oscillations of several longitudinal modes.

\section{Experimental Apparatus}

We measured the function $s(\lambda, \omega)$ for an $850-n m$ laser, by using a setup outlined in Fig. 1. A monochromator is here conveniently connected via two short lengths of step-index fiber (core diameter $\simeq 100 \mu \mathrm{m}$ ), whose spatial apertures actually replace the original input and output slits. The mode scrambler provides for the spatial launch stability required in bandwidth measurements [8]. Swept wavelength measurements were carried out using one modulation frequency at a time. Frequencies were chosen at $20 \mathrm{MHz}$ and in steps of $200 \mathrm{MHz}$ between 0 and $1200 \mathrm{MHz}$. At $f=0$ (nonmodulated source) we recorded the de output from a radiometer/detector in place of the APD.

In terms of the FWHM of a Gaussian approximation to the function $u$, the spectral resolution of the monochromator arrangement used above was of the order of $0.25 \mathrm{~nm}$. This is about 10 percent of the equivalent Gaussian FWHM of the measured function s. From (13) we find that the corresponding correction of $\sigma_{s}$ should be of the order of 1 percent, which we here consider to be negligible.

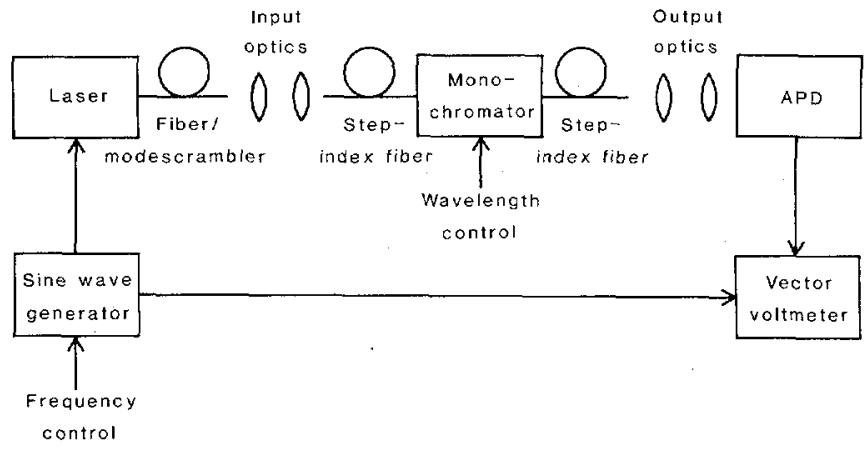

Fig. 1. Setup for measuring $s(\lambda, \omega)$.

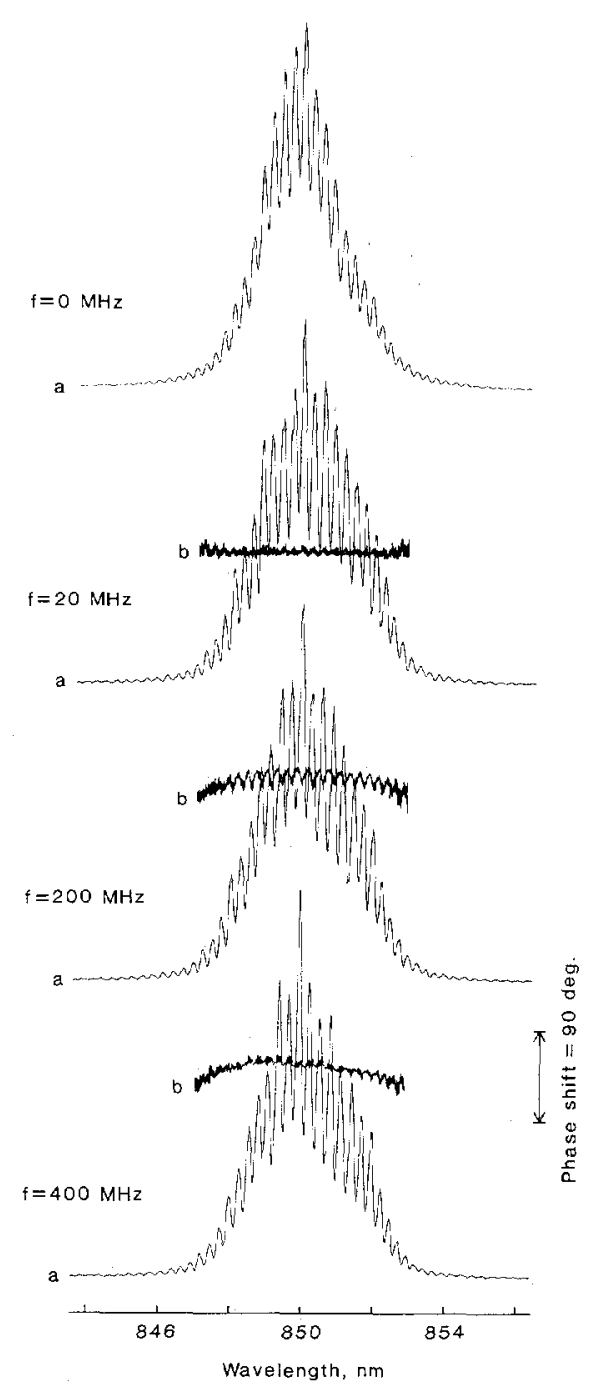

Fig. 2. The function $s(\lambda, \omega)$, measured at various modulation frequencies $f=\omega / 2 \pi$, amplitude $(a)$ and relative phase shift $(b)$.

\section{Results and Discussion}

Fig. 2 shows the measured amplitude and phase of the function $s$ at various modulation frequencies. The samples $s_{n}$ and $\theta_{n}$ were taken at the positions of the local amplitude peaks of $s$. Due to noise, phase data were acceptable only within a certain wavelength interval (as seen in the figure). Outside this interval, $\theta_{n}$ was retrieved by extrapolation of the accepted data. For each $n, s_{n}$, and $\theta_{n}$ could be estimated at other 


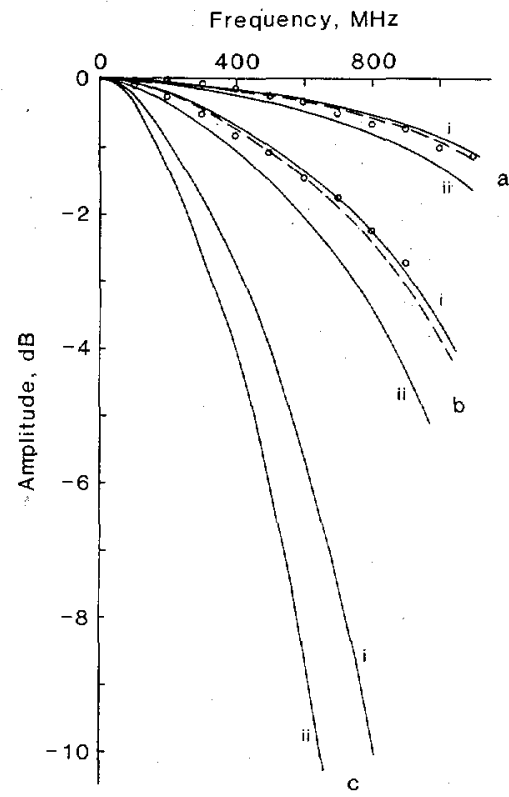

Fig. 3. Amplitude of the intramodal transfer function, calculated from (14) for (i) $\delta_{C}=-90 \mathrm{ps} \cdot \mathrm{km}^{-1} \cdot \mathrm{nm}^{-1}$, (ii) $-110 \mathrm{ps} \cdot \mathrm{km}^{-1} \cdot \mathrm{nm}^{-1}$ (solid curves); and $-92.5 \mathrm{ps} \cdot \mathrm{km}^{-1} \cdot \mathrm{nm}^{-1}$ (dashed curves). Fiber lengths: $z=1.02 \mathrm{~km}(a), 2.04 \mathrm{~km}(b)$ and $4.08 \mathrm{~km}(c)$. Dashed curves should be compared to data from a transmission experiment (rings).

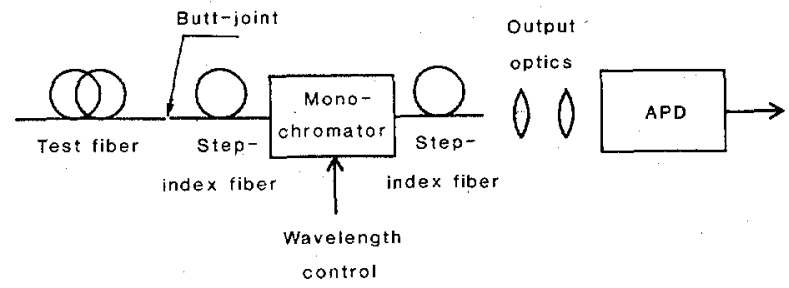

Fig. 4. Detail of setup for measuring the intermodal transfer function.

modulation frequencies than those of the measurements by linear interpolation along the frequency axis.

Intramodal transfer functions, calculated from (14), are depicted in Fig. 3 for three fiber lengths, 1.02, 2.04, and $4.08 \mathrm{~km}$. With reference to earlier results [2], we used two effective material dispersion coefficients, -90 and $-110 \mathrm{ps}$. $\mathrm{km}^{-1} \cdot \mathrm{nm}^{-1}$, in these calculations.

For a check of our results, we later tried to estimate the intramodal part experimentally (rings in Fig. 3). According to (6), we can obtain the intramodal part by dividing the total transfer function by the intermodal part. We thus measured the total function by a standard setup [9] while the intermodal function was obtained by inserting a monochromator, set to the strongest portion of the emitted spectrum, between the test fiber and the detector (Fig. 4). Due to the high insertion loss of the monochromator, intermodal measurements were restricted to fiber lengths of $2.04 \mathrm{~km}$ or less. Effects of finite spectral resolution (again of the order of $0.25 \mathrm{~nm}$ ) and spatial filtering of signals passing through the monochromator were here neglected. For comparison, the dashed curves in Fig. 3 were calculated from (14) using the available spectral data and a $\delta_{c}$ of $-92.5 \mathrm{ps} \cdot \mathrm{km}^{-1} \cdot \mathrm{nm}^{-1}$.

It is interesting to study the length dependence of the intramodal transfer function, on one hand predicted by our method,

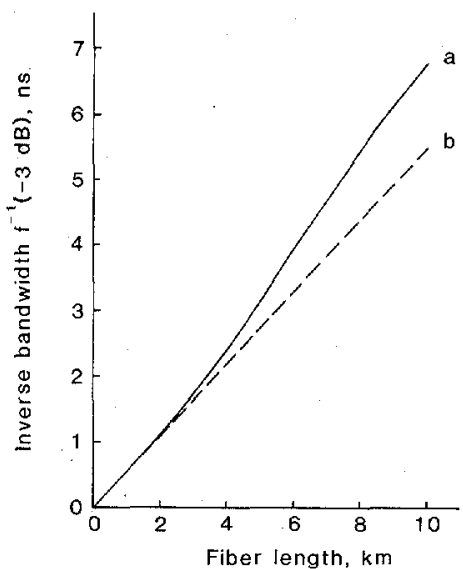

Fig. 5. Inverse intramodal bandwidth versus fiber length, rigorously calculated from $(14), \delta_{c}=-92.5 \mathrm{ps} \cdot \mathrm{km}^{-1} \cdot \mathrm{nm}^{-1}(a)$ and from a Gaussian fit to the spectrum of the nonmodulated source, $\delta_{c}=$ $-94.5 \mathrm{ps} \cdot \mathrm{km}^{-1} \cdot \mathrm{nm}^{-1}($ b $)$.

on the other by a more conventional method, where we fit a Gaussian function to the spectrum of the nonmodulated source (as depicted in Fig. 2) and apply (11). According to Fig. 5 , the -3 dB-bandwidths of the two predictions differ more than 20 percent for longer fiber lengths. Our more careful approach thus seems to be justified, even if we could not test predictions experimentally for lengths greater than $2.04 \mathrm{~km}$.

The conventional method assumes the same form for $s(\lambda, \omega)$ for all $\omega$. Our method, on the other hand, pays regard to the variation of $s$ with $\omega$. To qualitatively explain curve $a$ of Fig. 5 , we may for a moment consider both $T_{\text {intra }}$ and $s$ to be Gaussian in form. From the discussion associated with (9) and (10) we then find an inverse proportionality between $\Omega_{1}$, which we may call the $-3-\mathrm{dB}$ bandwidth of $T_{\text {intra }}$ with respect to the parameter $\Omega$, and $\sigma_{1}$, the FWHM of $s$ regarded at a fixed frequency $\omega_{1}$

$$
\sigma_{1}=K \Omega_{1}^{-1}=K / \delta_{c} \omega_{1} z_{1}
$$

where $K$ is a proportionality constant. This gives

$$
z_{1}=K / \delta_{c} \omega_{1} \sigma_{1}
$$

that is, with respect to baseband frequency, $T_{\text {intra }}$ has the bandwidth $\omega_{1}$ at the fiber length $z_{1}$.

We now consider a frequency $\omega_{2}<\omega_{1}$, say $\omega_{2}=0.5 \omega_{1}$, where the FWHM of $s$ is $\sigma_{2}$. Similarly, as before we obtain

$$
z_{2}=2 K / \delta_{c} \omega_{1} \sigma_{2} \text {. }
$$

if $\sigma_{2}>\sigma_{1}$, we get $z_{2}<2 z_{1}$. As an example we may here esti. mate the approximate Gaussian FWHM's of $s$ at 200 and 400 MHz from Fig. 2. We obtain $\sigma_{1} \simeq 0.9 \sigma_{2}$, which gives $z_{2} \simeq$ $1.8 z_{1}$, in good agreement with the corresponding points on curve $a$ of Fig. 5. Note in this specific case that it is the variation of spectral width more than a chirping effect that gives the nonlinear behavior of Fig. 5 .

\section{CONClusion}

We have derived the intramodal part of the transfer function in the case of a source spectrum that varies with modulation. As an application, we made calculations based on spectral measurements of a sinusoidally modulated, semiconductor $\mathrm{CW}$ 
laser. We here assumed the material dispersion to have been separately obtained. Particularly with regard to the length dependence of intramodal bandwidth, our predicted results were shown to differ substantially from those found by a conventional approach.

\section{APPENDIX A}

\section{Derivation of EquAtion (5)}

An intermodal transfer function may be defined from coupled power theory [5] as

$$
T_{\text {inter }}(\lambda, z, \omega)=\sum_{\mu=1}^{M} P_{\mu}(\lambda, z, \omega) / \sum_{\mu=1}^{M} P_{\mu}(\lambda, 0, \omega)
$$

where $P_{\mu}(\lambda, z, \omega)$ is a frequency component of the time dependent ensemble average mode power $p_{\mu}(\lambda, z, t)$. Output and input column vectors of these frequency components are, furthermore, related by

$$
\left(\begin{array}{c}
P_{1}(\lambda, z, \omega) \\
P_{2}(\lambda, z, \omega) \\
\cdots \\
P_{M}(\lambda, z, \omega)
\end{array}\right)=\boldsymbol{T}(\lambda, z, \omega)\left(\begin{array}{c}
P_{1}(\lambda, 0, \omega) \\
P_{2}(\lambda, 0, \omega) \\
\cdots \\
P_{M}(\lambda, 0, \omega)
\end{array}\right)
$$

where $\boldsymbol{T}$ is an $M \times M$ transfer matrix,

$$
\begin{aligned}
\boldsymbol{T}(\lambda, z, \omega) & =\exp [-\boldsymbol{H}(\lambda, \omega) z] \\
& =\boldsymbol{S}(\lambda, \omega) \exp [-\boldsymbol{R}(\lambda, \omega) z] \boldsymbol{S}^{-1}(\lambda, \omega) .
\end{aligned}
$$

$\boldsymbol{H}$ is an $M \times M$, symmetric and nonsingular matrix with entries

$$
H_{\mu \nu}= \begin{cases}a_{\mu}+\sum_{\eta=1, \eta \neq \mu}^{M} h_{\mu \eta}+i \omega \tau_{\mu}, & \mu=\nu \\ -h_{\mu \nu}, & \mu \neq \nu .\end{cases}
$$

The eigenvectors of $\boldsymbol{H}$ (and $\boldsymbol{T}$ ) are the columns of $\boldsymbol{S}(M \times M)$ while the eigenvalues of $\boldsymbol{H}$ are in the diagonal matrix $\boldsymbol{R}$ (also $M \times M)$.

Any eigenvalue $\rho$ of $\boldsymbol{H}$ can be found by solving

$$
\operatorname{det}[\boldsymbol{H}(\lambda, \omega)-\rho(\lambda, \omega) \boldsymbol{I}]=0
$$

where det $[\cdots]$ is a determinant and $I$ is the $M \times M$ identity matrix. By inserting (4) into (A4), we see that (A5) can also be written as

$$
\operatorname{det}\left\{\boldsymbol{H}\left(\lambda_{c}, \omega\right)-\left[\rho(\lambda, \omega)-i \omega\left(\lambda-\lambda_{c}\right) \delta_{c}\right] \boldsymbol{I}\right\}=0 \text {. }
$$

Any eigenvalue of $\boldsymbol{H}(\lambda, \omega)$ can thus be expressed as

$$
\rho(\lambda, \omega)=\rho\left(\lambda_{c}, \omega\right)+i \omega\left(\lambda-\lambda_{c}\right) \delta_{c} .
$$

It is easy to show [10] that $\boldsymbol{H}\left(\lambda_{c}, \omega\right)$ and $\boldsymbol{H}(\lambda, \omega)$ have the same eigenvectors; that is, $\boldsymbol{S}$ is independent of $\lambda$. Hence, from (A3) and (A7) we obtain

$$
\boldsymbol{T}(\lambda, z, \omega)=\boldsymbol{T}\left(\lambda_{c}, z, \omega\right) \exp \left[-i \omega\left(\lambda-\lambda_{c}\right) \delta_{c} z\right] .
$$

Equation (A2) then gives

$$
\begin{aligned}
P_{\mu}(\lambda, z, \omega)= & \exp \left[-i \omega\left(\lambda-\lambda_{c}\right) \delta_{c} z\right] \\
& \cdot \sum_{\nu=1}^{M} T_{\mu \nu}\left(\lambda_{c}, z, \omega\right) P_{\nu}(\lambda, 0, \omega)
\end{aligned}
$$

$T_{\mu \nu}$ being the $\mu \nu$ th entry of $\boldsymbol{T}$.
Since the mode excitation may be influenced by spectral variations we can write

$$
P_{\mu}(\lambda, 0, \omega)=g(\lambda, \omega) P_{\mu}\left(\lambda_{c}, 0, \omega\right) .
$$

$g(\lambda, \omega)$ is here a mode independent function of $\lambda$ and $\omega$. Of course, $g\left(\lambda_{c}, \omega\right) \equiv 1$ irrespectively of $\omega$. By inserting (A10) into (A9) we arrive at

$$
P_{\mu}(\lambda, z, \omega)=\exp \left[-i \omega\left(\lambda-\lambda_{c}\right) \delta_{c} z\right] g(\lambda, \omega) P_{\mu}\left(\lambda_{c}, z, \omega\right) .
$$

If we use (A11) together with (A1), we may now easily obtain (5).

\section{ACKNOWLEDGMENT}

The author would like to thank G. W. Day for his assistance in the course of this work.

\section{REFERENCES}

[1] T. Tanifuji and M. Ikeda, "Simple method for measuring material dispersion in optical fibers," Electron. Lett., vol. 14, pp. 367$369,1978$.

[2] D. L. Franzen and G. W. Day, "Measurement of propagation constants related to material properties in high-bandwidth optical fibers," IEEE J. Quantum Electron., vol. QE-15, pp. 1409-1414, 1979.

[3] F. T. Stone, "Problems in bandwidth measurement and a suggested solution," J. Lightwave Tech., vol. LT-1, pp. 207-214, 1983.

[4] J. V. Wright and B. P. Nelson, "Pulse compression in optical fibres," Electron. Lett., vol. 13, pp. 361-363, 1977.

[5] P. M. Rodhe, "An experimental study of a matrix transfer function for an optical fibre," in Proc. 7th European Conf. Optical Communication, (Copenhagen, Denmark), 1981.

[6] M. V. Klein, Optics. New York: Wiley, 1970, pp. 220-225.

[7] See for example: H. J. Blinchikoff and A. I. Zverev, Filtering in the Time and Frequency Domains. New York: Wiley, 1976, pp. $415-417$.

[8] W. F. Love, "Novel model scrambler for use in optical fiber bandwidth measurements," Tech. Dig. Opt. Fiber Commun., Washington: Optical Society of America, 1979.

[9] G. W. Day, Measurement of Optical Fiber Bandwidth in the Frequency Domain, NBS TN-1046, Washington: U.S. Government Printing Office, 1981.

[10] G. Strang, Linear Algebra and its Applications. New York: Academic, 1980 , ch. 5 .

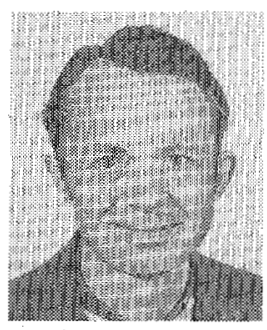

Peder M. Rodhe was born in Stockholm, Sweden, on May 13, 1945. He received the M.S. degree in Physics from the University of Stockholm, in 1973 and the Lic.Techn. and Ph.D. degrees in Optics and Wave Theory from the Royal Institute of Technology, Stockholm, in 1982 and 1984 , respectively.

From 1975 to 1979 he was engaged in research and development of optical fibers at Ericsson in Stockholm. After that he joined the Fiber Measurement Group at Sieverts Kabelverk $A B$, Sundbyberg and from 1980 to 1982 he also was an assistant at the Institute of Optical Research, Stockholm. On a fellowship from the Wallenberg Foundation he became a guest worker at the National Bureau of Standards, Boulder, CO, from 1982 to 1984, also joining the University of Colorado as a visiting research associate. Very recently, he obtained a position as an engineering scientist at the Photonics Research Group at AMP Incorporated, Harrisburg, PA. 